\title{
An Overview of Microneedles: Types, Materials, Processes, Emerging Approach and
}

\section{Applications in Dermatology}

Snehal S. Patil*, Nikita S. Patil, Umesh C. Suryawanshi, Mr. Rohan R. Vakhariya, Dr. S. K. Mohite

Rajarambapu College of Pharmacy, Kasegaon, Dist. Sangli, Maharashtra, India

Email ID: patilsnehal5121@gmail.com

\begin{abstract}
:
Drug delivery through the skin by transdermal patches has a long history. Subsequent growth of transdermal science proved prominent utility of transdermal systems meant for passive diffusion of the drug. It was followed by the development of Iontophoresis and Sonophoresis based transdermal delivery systems. Microneedle array has now caught attention of the investigators owing to its immense utility in transdermal delivery of very large molecules with ionic and hydrophilic nature. In this technical note, we present the current scenario, applications, and recent advances in microneedle array-based delivery of the most critical molecules through the skin. The application of microneedle has widely been investigated, and these technologies are being developed for the delivery of bio-therapeutics, biomacromolecules, insulin, growth hormones, immunobiologicals, proteins, siRNA and peptides. Potential of microneedles to transform the global transdermal market is highlighted in terms of the success rate of the microneedle technologies in clinical trials reaching to the global market. The arrival of the commercial microneedle-based products in the market is highly anticipated as they have potential to portray remarkable impact on clinical medicine in near future.
\end{abstract}

Keywords: Microneedles, transdermal, drug delivery, technologies. 


\section{Introduction:}

The skin is the largest organ in the human body in terms of body weight and it plays an important role, not only in its shield-like external defense function but also in the regulation of temperature and water. ${ }^{1}$ Being the outermost layer of the body, the presence of skin protects the rest of the body from direct exposure to the various microorganisms and pathogenic factors present in the external environment. The architecture of skin has been recognized as three layers perpendicular to the skin surface: epidermis, dermis and subcutis (Fig. 1). The epidermis and dermis layers bind tightly to each other on the surface of the skin, forming a complete envelope structure, and are separated from deeper tissue by the subcutis. The epidermis is the most supercial and biologically active of these layers, as the basal layer of the epithelium (stratum basale) is constantly renewing. ${ }^{2}$

The stratum corneum (SC) also forms part of the epidermis and its normal thickness is approximately $100 \mathrm{~mm}$. The $\mathrm{SC}$ is a major anatomical component of the skin's barrier function and of its regulation of water. In some immune skin diseases, the skin is the target tissue of the immune response attack. Moreover, the human skin itself forms a "skin immune system", which consists of many different types of cell including Langerhans cells, T lymphocytes, keratinocytes, macrophages, and dendritic cells. One of the basic functions of human skin is absorption, and the realization of this function mainly depends on the existence of the SC. In dermatologic conditions, the absorption function of the skin is the principle method by which topical drugs are used to treat diseases. However, the absorption function of the skin is limited, and is an obstacle to topical drug absorption by the skin. It must be mentioned here that there is an easily overlooked function of skin - aesthetic appreciation. A particularly interesting claim is that people "wear their health" on their skin. ${ }^{3}$ The smoothness, integrity, and evenness of human skin can greatly influence a person's appearance from a visual perspective. There is no wonder why people care about skin conditions and how skin problems are solved in numerous ways. Therefore, the optimization of skin treatment is particularly important. With skin being the largest organ of the human body, skin diseases are experienced by a wide range of patients globally. Taking psoriasis as an example, $2-3 \%$ of the world's population suffers from this condition, $80-90 \%$ of who have plaque psoriasis; more than one-third of them present with the moderate-severe form of the disease. ${ }^{4}$

Psoriasis has a significant impact on quality of life; visible plaques, desquamation, and itching are the most common and concerning symptoms and signs. The current treatment for psoriasis, although effective, remains unsatisfactory in the long term and treatment challenges still exist. Drug administration through the gastrointestinal tract or circulatory system sometimes increases drug consumption or reduces local drug concentration per unit area of skin. Topical cream spreads only on the skin surface. It is reported that only 10-20\% or less of total drug loaded in cream is permeated through the skin. To solve this problem, dermatologists are increasingly turning their attention to transdermal drug delivery systems (TDDSs).In this field, emerging TDDSs using microneedles have developed rapidly in recent decades. Microneedle drug delivery systems have been developed and are considered to be hybrids of the problematic hypodermic needle and transdermal patch. Microneedle systems comprise a base plate with microneedles arrays perpendicular to it. The microneedles patch (MNP) is a more accurate definition of microneedles loaded with drugs. 
Once the microneedles pierce the skin, the loaded drug has greater opportunity to directly contact the epidermis or dermis without the challenge of a barrier. The procedure makes drug delivery much easier, bypassing the main obstacle in a "smart" way. As a convenient invasive delivery device, the properties of microneedle treatment make it an excellent choice, with the capacity to transport various drugs or other substances suitable for this method to the human body. This review aims to describe the characteristics and advantages of several accepted microneedle types and their potential in psoriasis treatment, with particular attention to the microneedle patch (MNP).

\section{Micro needle-Based Transdermal Delivery Approaches:}

The success of MNA-based drug delivery is determined by critical issues, mainly concerning the design of the MNA (shape, size, geometry, and manufacturing materials and processes) and the type of active substance delivered. The different strategies can be classified as: "poke and patch", "poke and flow", "coat and poke", and "poke and release".

\subsection{Solid Microneedles for "Poke and Patch":}

The "poke and patch" approach consists in the use of solid MNA to perforate the skin, creating micro channels that reach the deepest layers of the epidermis. This method significantly improves the passive transport of drugs through the skin, since the main barrier to permeability, the stratum corneum, is disrupted ${ }^{6,7}$. This approach presents two steps: First, the MNA are used to pierce the epidermis and are subsequently removed; and second, the drug is applied in a conventional dosage form (solution, cream, or patch), which works as an external drug reservoir (Figure 1). Its simplicity, from the technological point of view, makes it highly attractive, especially for its easy application in a clinical setting. However, this technique is not exempt of controversy and presents several disadvantages. One of the main drawbacks is that the micro pores remain open only for a limited time, potentially stopping the delivery of the active substance prematurely. It has been reported that all microneedle treated sites recovered their barrier properties within $2 \mathrm{~h}$. Nevertheless, this period can be extended up to three days under occlusive conditions by using formulations like patches or tapes ${ }^{8}$, although the risk of infection increases considerably in these conditions ${ }^{9}$.

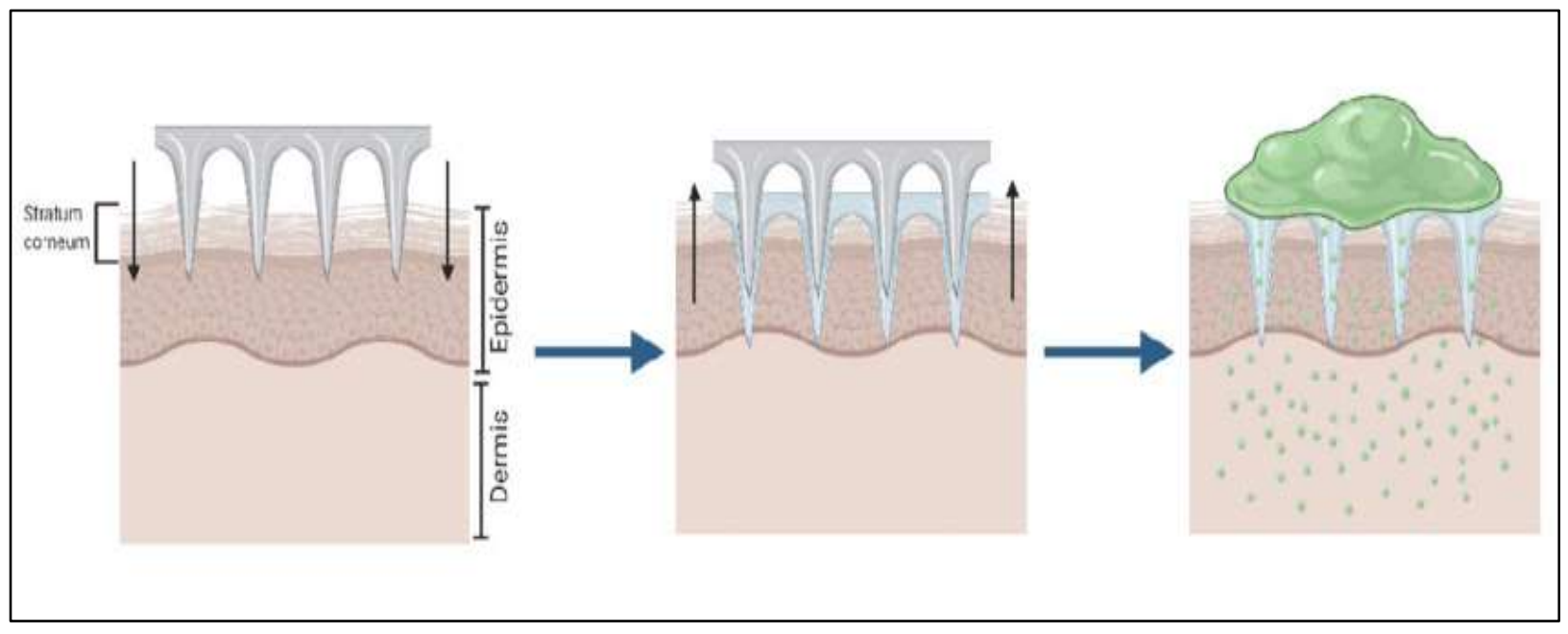

Figure No. 1 Schematic representation of the "poke and patch" approach with solid microneedle arrays (MNA) 


\subsection{Coated Microneedles for "Coat and Poke":}

Another approach with solid MNA is the "coat and poke" technique which requires the coating of the solid microneedles' surface with a drug or vaccine-loaded formulation. This strategy allows drug diffusion from the coating surface to the deeper epidermal layers after MNA insertion (Figure 2) ${ }^{10}$. Certain issues, mainly related to the coating, limit the usefulness of this approach. For instance, the amount of drug which can be encapsulated in the coating layer is relatively low. Besides, the coating's thickness can decrease the sharpness of the microneedles and influence their ability to perforate the skin. Despite this, coated MNA have shown great efficiency in vaccination, since the antigen dose needed to trigger an immune response is usually in the range of nano or micrograms ${ }^{11}$.

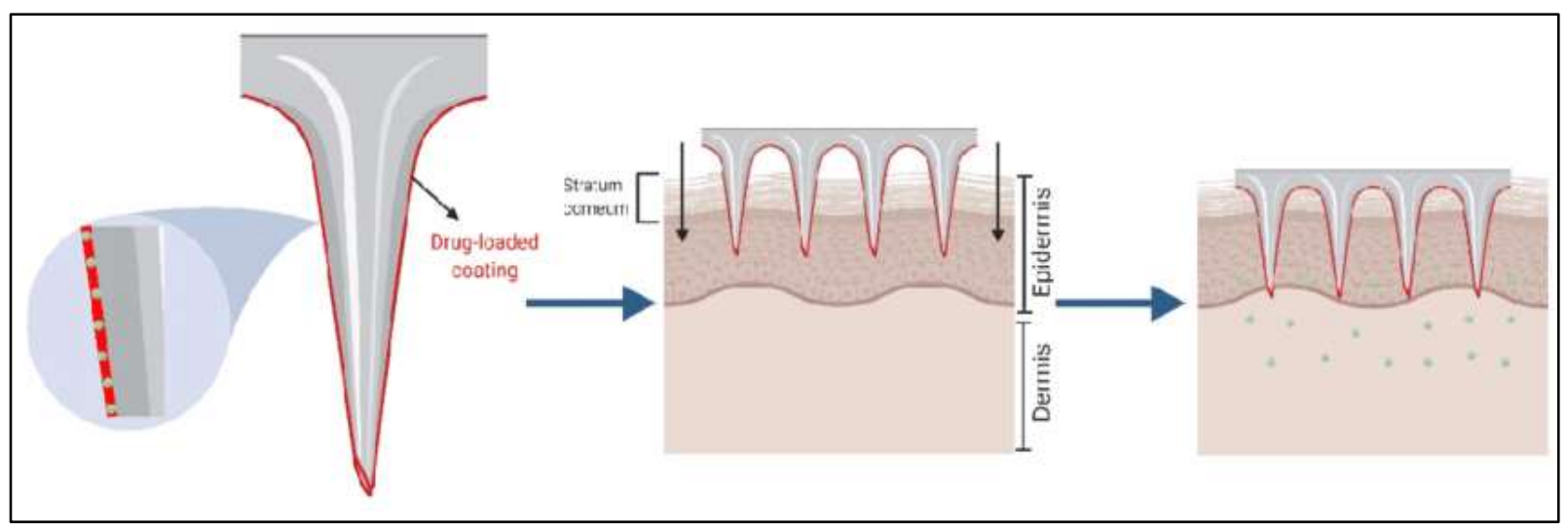

Figure No. 2 Schematic representation of "coat and poke" approach

\subsection{Dissolving and Hydrogel-Forming Microneedles for "Poke and Release":}

Dissolving MNA can be made of a range of water-soluble and biodegradable materials in which the drugs can be loaded and released as the MNA dissolves after insertion (Figure 3). The improvement seen in this approach in comparison with the "poke and patch" is that dissolving microneedles can maintain controlled drug release over a longer period of time, by controlling the dissolution rate of the formulation used as the MNA matrix. Another advantage is that it reduces the drug administration process to one step, as the MNA are able to pierce the skin and are kept inserted until complete dissolution ${ }^{12,13}$. Besides, dissolving MNA avoids the generation of sharps waste, minimizing the cost related to its management and reducing needle-stick injuries. On the other hand, the drawbacks include a limited drug loading and a potentially lower ability to perforate the stratum corneum.

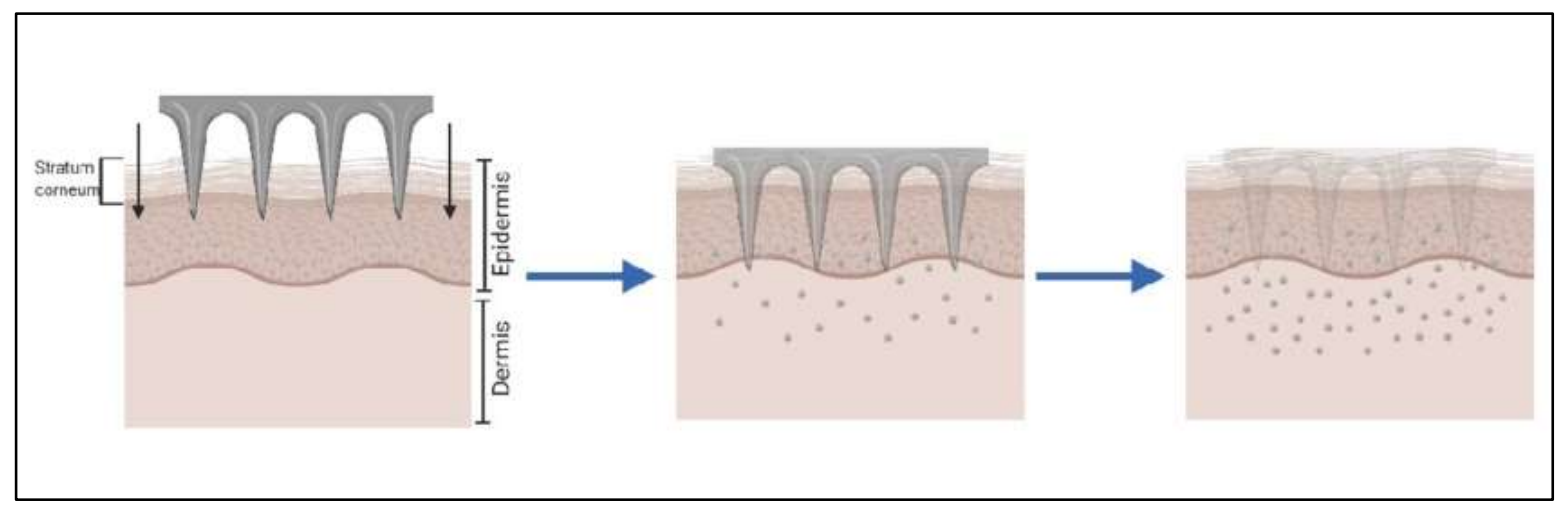

Figure No. 3 Schematic representation of "poke and release" approach 
Rapidly separating MNA was designed as a hybrid between coated and dissolving MNA (Figure 4). The aim is to insert in the skin a drug-loaded water-soluble matrix encapsulating the drug, coupled with a solid MNA composed of an insoluble polymer. This second array helps the insertion of the soft matrix that remains in the skin, while the solid MNA can be easily removed afterwards. As an evolution of these MNA, more sophisticated designs have been developed. The insertion of air bubbles in the MNA structure, between the tips and the patch base, enables the easy and rapid separation of the microneedles tips from the backing structures after insertion, leaving the tips in the skin and generating non- sharp wastes. ${ }^{14}$

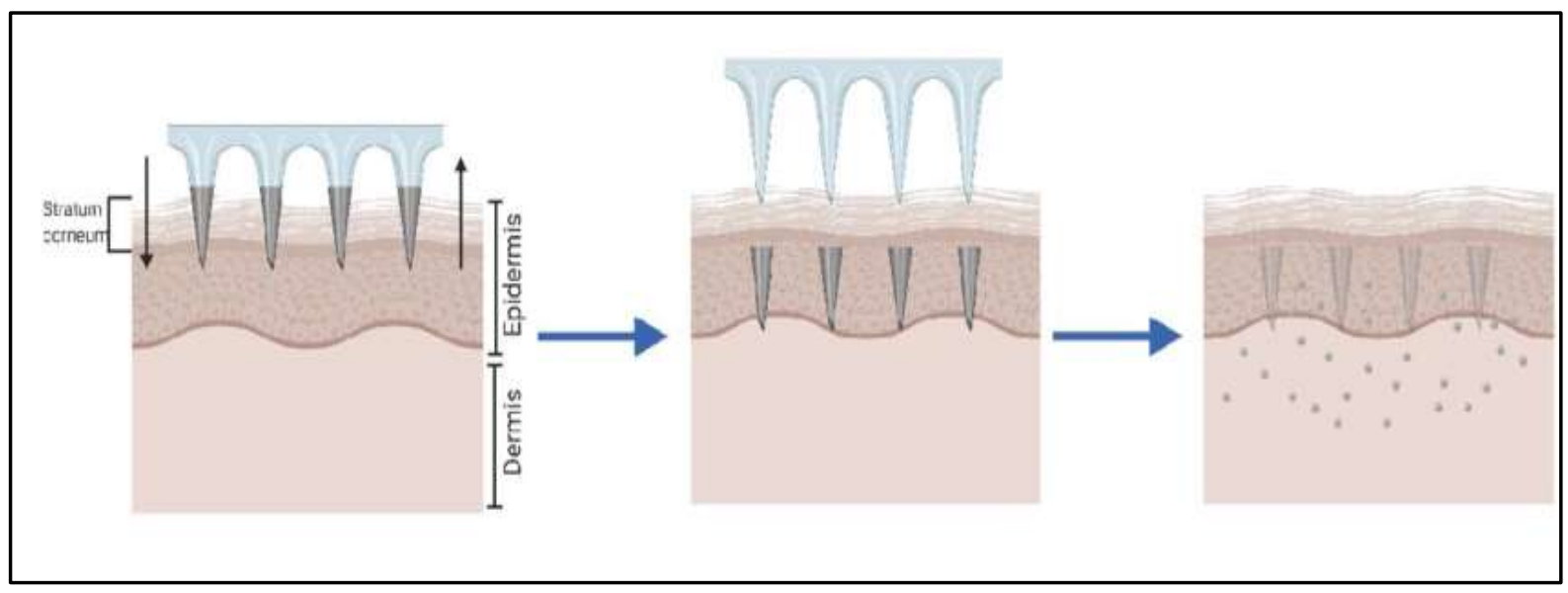

Figure No. 4 Schematic representation of rapidly separating MNA

As an alternative to "poke and patch" approaches, hydrogel-forming MNA or swellable MNA have been developed (Figure 5). The aim of these devices is to imbibe skin interstitial fluid upon insertion to form continuous, unblockable micro channels amongst dermal capillaries. This approach allows the release of less potent drugs contained in an attached patch type drug reservoir.

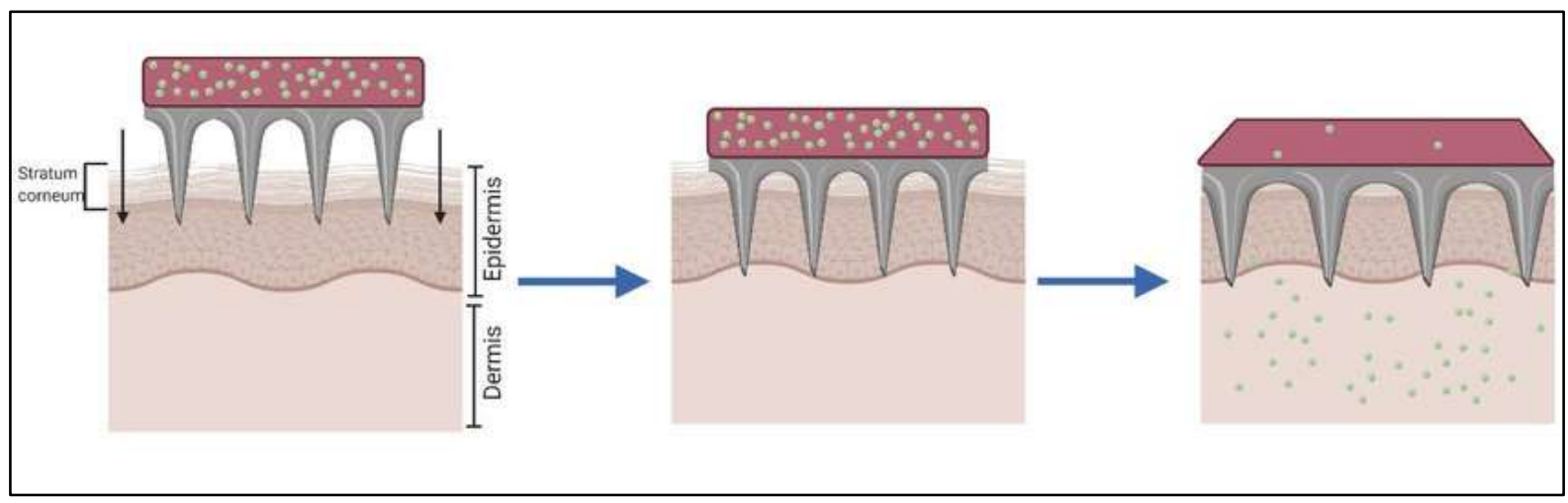

Figure No. 5 Schematic representation of hydrogel-forming or swelling MNA

\subsection{Hollow Microneedles for "Poke and Flow":}

The "poke and flow" approach was conceived to introduce a drug solution into the skin mimicking hypodermic injections while overcoming their limitations ${ }^{15}$. In this approach, the microneedles play a similar role to hypodermic needles, through which drug formulations are administered after skin perforation (Figure 6) Due to their micrometric size, their manufacturing process is difficult and expensive, requiring significant technological 
resources. By contrast, thanks to the shorter size of these needles, the average patient's acceptance of this approach is higher than that of traditional injections.

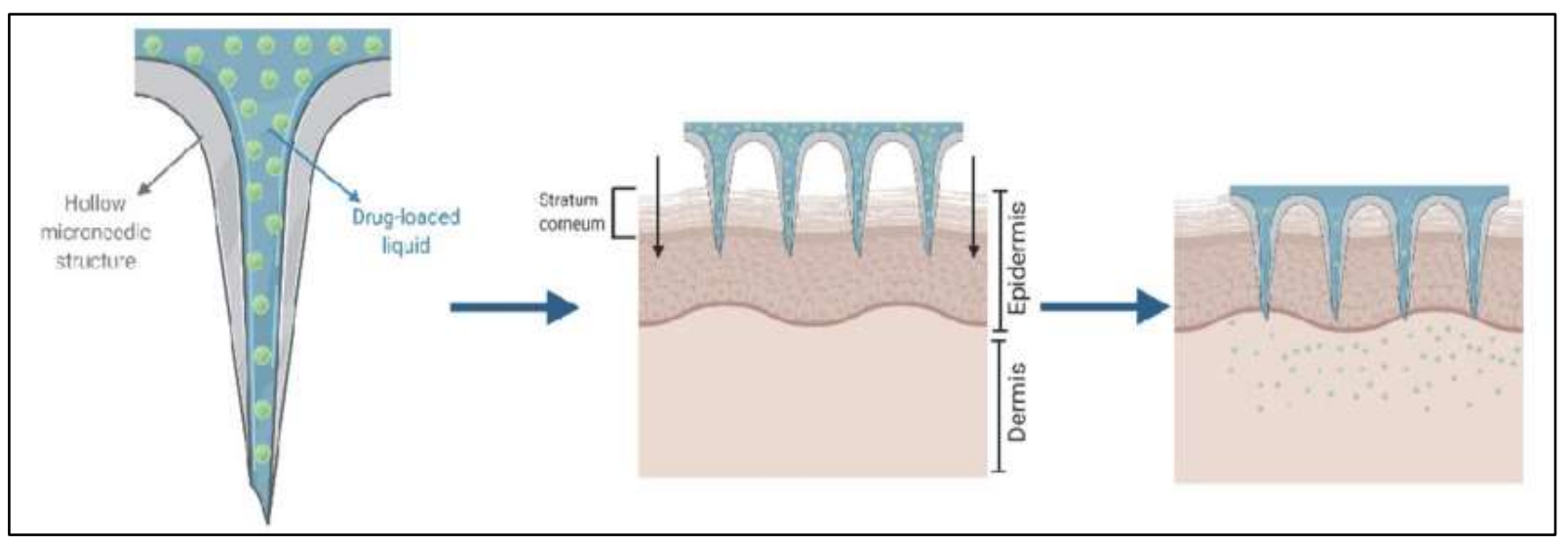

Figure No. 6 Schematic representation of "poke and flow" approach

\section{Methods of Drug Delivery:}

\subsection{DNA Vaccine delivery:}

The cells of Langerhans present in the skin serve as the first level of immune defence of the body to the pathogens invading from the environment. These cells locate the antigens from the pathogens and present them to $\mathrm{T}$ lymphocytes, which in turn stimulate the production of antibodies. ${ }^{16}$ The arrays were dipped into a solution of DNA and scrapped multiple times across the skin of mice in vivo. Expression of luciferase reporter gene was increased by 2800 fold using micro enhancer arrays. In addition, microneedle delivery induced immune responses were stronger and less variable compared to that induced by the hypodermic injections. Similar results were obtained by researchers at Beckett- Dickinson ${ }^{\mathrm{TM}}$ in an animal study for antibody response to HepB naked plasmid DNA vaccine. This approach has a potential to lower the doses and the number of boosters needed for immunization.

\subsection{Desmopressin delivery:}

Some researchers examined the use of microneedles to deliver desmopressin, a potent peptide hormone used in the treatment of nocturnal enuresis in young children, as well as for the treatment of diabetes insipid us and haemophilia A. ${ }^{17}$ Microneedles were coated by an aqueous film coating of desmopressin acetate on titanium microneedles of length $200 \mu \mathrm{m}$, a maximal width of $170 \mu \mathrm{m}$ and a thickness of $35 \mu \mathrm{m}$. Microneedle patch was inserted into the skin with the help of an impact applicator. A target dose of $20 \mu \mathrm{g}$ of desmopressin was delivered to hairless guinea pig from $2 \mathrm{~cm}$ square microneedle array within 15 minutes. This study demonstrated that the transdermal delivery of desmopressin is safe and efficient alternative to the currently available routes of administration

\subsection{Local Tissue Delivery:}

Conventional drug delivery often involves administering medication systemically, thereby treating the desired region of the body, but also exposing other parts of the body to the drug, which can have detrimental effects. Drug delivery targeted to a precise region in the body can reduce side effects, minimize the dose of a costly drug, or provide a means of delivery to a location that is difficult to treat ${ }^{18}$. Two novel devices have been discussed that deliver drugs 
to specific regions of tissue inside the body. Micro fabricated neural probes have been used to deliver drugs into neural tissue of guinea pigs in-vivo while simultaneously monitoring and stimulating neuronal activity. Microprobes have also been inserted across vessel walls of normal and atherosclerotic rabbit arteries in-vitro.

\subsection{Systemic Delivery:}

In recent years, biotechnology has produced a battery of sophisticated and potent drugs. However, methods to effectively deliver these drugs into the body have limitations ${ }^{19,20}$. Oral delivery of the new protein based, DNA-based and other therapeutic compounds is generally not possible owing to drug degradation in the gastrointestinal tract/ or elimination by the liver. The usual alternative to oral delivery is via injection, either directly into the bloodstream or into tissues (e.g. subcutaneous or intramuscular injection). Although injection effectively delivers drug in large quantities, it has significant limitations, such as pain and trauma caused by the needle, failure to provide convenient controlled or sustained release, and the need to expertise to perform an injection. To overcome these limitations microneedles have been designed in such a way that by decreasing the size of hypodermic needles, insertion pain and tissue trauma experienced by patients can be reduced. Also, the combination of these needles with micro pumps and other devices can yield more sophisticated needles that can potentially deliver drugs in a more controlled manner.

\section{Evaluation of microneedles:}

\subsection{Characterization of microneedle geometry:}

Scanning electron microscopy can be used to determine the base radius, tip radius and wall thickness of the microneedles. Interfacial area (i.e. the effective area of contact between the needle and the skin) can be calculated in two ways: (1) the annular surface area, Aa; at the needle tip

$$
\mathrm{A}_{\mathrm{a}}=\pi\left(\mathrm{r}_{\mathrm{t}} \mathrm{t}-\mathrm{t}^{2} / 4\right)
$$

and the full cross-sectional area, Af; at the needle tip

$$
\text { Af }=\pi r_{t}^{2}
$$

Needle wall angle, a, is calculated as

$$
\alpha=\tan ^{-1}\left(\mathrm{r}_{\mathrm{b}}-\mathrm{r}_{\mathrm{t}} / \mathrm{h}\right)
$$

Where, $\mathrm{rt}$ is the outer radius of the microneedle tip, $\mathrm{rb}$ is the outer radius at the needle base, $\mathrm{t}$ is the wall thickness and $\mathrm{h}$ is the height. ${ }^{21}$

\subsection{Functional capacity test:}

Evaluated the functional capacity of microfluidic lumens using a custom fluidic test setup. ${ }^{22}$ The test setup consisted of a syringe pump system with a dye-filled syringe, a polymer tube and microneedle array. This syringe pump system was used to examine the formation of the microneedle lumens by allowing dye to flow from the syringe to the microneedle orifice. Microscopic inspection of the microneedle tips and the base plate during the microfluidic characterization can be used to detect cracks in the base plate and passage continuity.

\subsection{Measurement of insertion force into human skin:}


A displacement-force test station was used to measure the force applied to a needle, needle position and skin resistance during the sequence of the needle's translation, deflection of tissue around the needle and insertion into the skin of human subjects. ${ }^{23} \mathrm{~A}$ drop in electrical resistance of the skin was used to identify needle penetration since visual observation of needle insertion was extremely difficult. The electrical resistance of skin's outermost layer, the stratum corneum, is much greater than deeper tissues; therefore the resistance of the skin drops dramatically as soon as a needle penetrates.

\subsection{Margin of safety:}

The margin of safety as the ratio between the force required for piercing the stratum corneum and the force at which microneedles broke. ${ }^{24}$ They hypothesized that if the ratio is $<1$ then microneedle array can be used in biomedical application. They checked margin of safety for silicon microneedles using computerized apparatus. For compressive failure force measurement, Enduratec station was used in which microneedles were placed between punch and load cell. An appropriate margin of safety was found for sample silicon microneedle arrays.

\subsection{Measurement of fracture force:}

The force required for mechanical fracture of a microneedle was tested by employing an axial load test station that drove the microneedle against a flat block of aluminium at a rate of 0.01 $\mathrm{mm} / \mathrm{s}$ until a preset displacement of $500 \mathrm{~mm}$ was reached. Microneedles were attached to the testing surface using adhesive tape around the base of the needle. Microneedle fracture was observed through an attached microscope to evaluate the mode of failure. The force and displacement data were used to quantitatively determine the fracture force.

\subsection{Penetration/diffusion test:}

\subsubsection{In-vitro and ex-vivo test:}

In-vitro/ex-vivo tests are performed on isolated animal/ human dermatome skin to study penetration or diffusion of drug from a dosage form to its site of application. These tests can also be used to compare the depth of penetration of the molecule. Use of confocal laser scanning microscopy (CLSM) to demonstrate the depth of penetration of Rhodamine $\mathrm{B}$ in human dermatome skin using microneedles of $150 \mathrm{~mm}$ length. ${ }^{25}$ They reported the concentration of the dye to be very weak below $80 \mathrm{~mm}$ depth. They also evaluated the penetration of model drug using Franz diffusion cell across the microneedle-treated and untreated skin and reported enhancement in penetration by 104 to 105 times with use of microneedles. Similar observations were also reported by other researchers. On the other hand penetration of 1-ascorbic acid was not significantly increased through microneedletreated skin as compared with untreated skin. They punctured the skin more than once to demonstrate the effect of application frequency of microneedles. They concluded that permeation of 1-ascorbic acid was increased by 10.54-fold only after insertion of microneedles in four directions nine times. Scientists investigated penetration of microneedles both in vitro and ex vivo by injecting Rhodamine B dye. For in-vitro testing $1 \%$ agarose gel was used and for ex-vivo testing, chicken breast flesh, laboratory mouse and an anaesthetized rabbit were used. The Rhodamine B easily penetrated across the $1 \%$ agarose gel and chicken breast flesh; the penetration in $1 \%$ agarose gel can clearly be viewed. 


\subsubsection{In-vivo test:}

For a transdermal drug delivery system, it is practically impossible to predict the skin permeability of formulations using in-vitro experiments alone. Significantly different results might be observed while performing in-vivo study. ${ }^{26}$ They reported that the transport of insulin through rat skin was found to increase by 10-20 times in an in-vitro study, while during their in-vivo study, microneedles failed to deliver drug systemically. Thus, along with in-vitro/ex-vivo testing, in-vivo tests should always be performed. If correlation is established between ex-vivo and in-vivo models, the drug development process could be made more economic and shorter. Penetration of fluorescent dye completed through human skin after microneedle application with the help of confocal laser scanning microscopy (CLSM). Further, used optical coherence tomography (OCT) for imaging of tissue structure. Both these methods provide tissue imaging without the need for tissue pre-treatment or removal. Penetration of methylene blue dye at the microneedle treated application site in domestic swine after removal of array. A hilltop chamber was attached to the application site. After a 10-min staining period, the chamber was removed followed by washing with distilled water and observed for penetration. Analysis of plasma concentrations was also adopted by a few scientists to compare the microneedle technique with other TDDS. Researcher measured the maximum blood fluxes and time taken to reach maximum flux in healthy human volunteers for comparison of pointed microneedle, hollow microneedle and topical application. They found that the time required to reach maximum blood flux was significantly decreased after administration of methyl nicotinate by pointed and hollow microneedles as compared with topical administration. Similarly higher plasma concentration of ketoprofen was achieved in rats with microneedles in comparison with a topical gel formulation alone. They found increment in both $\mathrm{C}_{\max }$ and AUC of ketoprofen after application with microneedles. In-vivo delivery of insulin using microneedles in rats and after that examined the resultant blood glucose level. This was decreased by $80 \%$ with the use of microneedles. Administration of radio labelled albumin intradermally in mice using a microneedle-based miniature syringe. They found a significant amount of radioactivity in mouse skin even after wiping off the site of application with wet tissue, which was indicative of successful delivery of protein (albumin).

\section{Applications:}

Skin is suitable for gene and oligonucleotide delivery because it is well characterized at the cellular as well as the molecular level. The microneedle delivery system can be used for treatment of various genetic diseases related to skin, various types of malignancies and infectious diseases, and for immunization. A dense array of very sharp pyramidal microneedles was used to deliver gene into cells. Microneedle delivery of gene is better than a microinjection technique because many cells can be treated at once. Thus microneedles can be used to deliver bioactive agents systematically as well as locally. Research could focus on antiviral, antidiabetic, genetic, oncological, anti-osteoporosis, vaccine, dermatological, etc., areas for bioavailability improvement by developing microneedle-based transdermal drug delivery systems.

\subsection{Immunobiologicals:}

Conventionally immunobiologicals are administered through a needle via the subcutaneous, intramuscular or intradermal route for prevention of infectious diseases. However the 
conventional vaccination procedure suffers from drawbacks like needle phobia and the pain associated with insertion of needle into the skin. Research has focused on development of needle-free vaccination like liquid jet injectors, powder injectors, thermal ablation and microneedles. Microneedles have an edge over the other methods due to lack of pain, self administration and quick delivery of vaccine. Combination vaccination is one of the ways to reduce the number of injections to be administered; 'DPT' is a well known example, used to prevent infection of diphtheria, pertussis and tetanus. However to develop such a formulation is a challenge as the physical, chemical and biological interactions between the vaccine components may have a detrimental effect on vaccine safety or efficacy. Physical and chemical interaction as well as adverse effect on the biological activity of each component was not observed by researchers. Conventional liquid vaccines require cold conditions during transportation and tend to have a short shelf life. The stability of vaccines at high temperature as well as maintenance of antigenicity in coated microneedles. ${ }^{27}$

\subsection{Bioactive macromolecules (biopharmaceuticals):}

Insulin, heparin, and growth hormones are not administered orally due to proteolytic degradation and hindered absorption. The majority of commercially available biopharmaceuticals are administered via the parentral route and hence a suitable non-invasive route is desirable. Administered macromolecules with varying molecular weight across human dermatomed skin using microneedles. ${ }^{28}$ They revealed that microneedle arrays enhanced the transport across dermatomed human skin for both low and high molecular weight compounds.

\subsection{Drugs:}

Very few drug molecules possess the necessary physicochemical properties to cross the skin barrier and even if the drug can cross the barrier, drug delivery rate via the transdermal route is very low. Physicochemical properties like hydrophilic-lipophilic balance, solubility, molecular weight, etc., govern the transport of a drug through the skin and also the rate of transportation. These challenges can be overcome by use of microneedles. Highly hydrophilic drug formulations like PEGylated naltrexone or hydrophobic formulations of drugs like ketoprofen, show a many-fold increase in area under the curve (AUC) and maximum drug concentration $(\mathrm{Cmax})$ as compared with conventional cream or gel formulations. The need for penetration enhancers, which may induce irritation, can be eliminated by the use of microneedles. In photodynamic therapy for cancer, a combination of photosensitive drug and light is used to destroy the selected cells via generation of highly cytotoxic singlet oxygen. Topical administration of such high-molecular-weight photo sensitizer drugs results in poor bioavailability. Their accumulation in normal cells precludes their intravenous administration. Worked on microneedle-mediated intradermal delivery of 5-aminolevulinic acid and transdermal delivery of meso-tetra ( $\mathrm{N}$-methyl-4-pyridyl) porphine tetra tosylate using silicon microneedles. ${ }^{29,30}$

\subsection{Phlebotomy:}

Phlebotomy is the withdrawal of blood for diagnostic purpose. Analysis of blood samples for specific blood constituents helps in the diagnosis of a disease. Some diseases like diabetes require frequent monitoring of blood for estimation of glucose concentration or disease severity. Painless hollow microneedle-based micro sampling can be used instead of 
traditional methods for glucose estimation. ${ }^{31}$ They resolved problems in microneedles, like bending and clogging, by a combination of design and fabrication processing to achieve optimal tip shape, opening size, needle height and needle area density. Blood samples are generally collected from capillaries by pricking the skin or from veins using evacuated collection tube, depending on the volume of blood required for analysis. These methods are associated with disadvantages such as excessive bleeding, infection, scarring, fainting or feeling light-headed. People may hesitate to give blood due to fear of needles and the moderate pain associated with the procedure. Hence painless blood sampling using microneedles can be a very good alternative to hypodermic needles. It can be concluded that the design and dimensions of microneedles used for blood sampling are the most important parameters to be precisely selected for easy penetration to a particular depth in the skin without bending, damage to nerves situated deep inside dermis, and tissue trauma. The device can be used anywhere on the skin for sampling, therefore there is the need to place it on a large visible vein or on the finger tip. This approach can be used for monitoring of therapeutic drug levels too. ${ }^{32,33}$

\subsection{Diagnosis:}

Hollow microneedles can be used to withdraw fluid from tissue or blood which can be subsequently analyzed to check status of diseases like cancer, diabetes and many more. Hollow microneedles, along with quantum dots, help in medical diagnosis. Quantum dots are nano scale crystals with a light-emitting property. The multi photon microscopy method could rapidly diagnose cancers or other medical problems. Evaluated the influence of geometry and design of microneedles on the piercing ability (through the skin) and capillary movement of interstitial fluid. ${ }^{34}$

\subsection{Cosmetic products:}

Generally, only minor fractions (maximum $0.3 \%$ ) of the active substance present in a cream, gel or lotion can penetrate deeply into the skin. This means that the majority of an active ingredient, about $99.7 \%$, is wasted. Derma rollers and stamps are available on the market for treatment of skin problems as well as to improve looks. Clinical Resolution Laboratory markets MTS Derma roller, a cosmetic aid possessing needles that penetrate the skin up to a depth of $0.2-0.3 \mathrm{~mm}$. The product contains 200 very fifine stainless-steel needles to pierce the epidermis, creating a micro-channel effect. Clinical studies from various countries have proven that therapeutic serum absorption is increased by as much as 1000 times when applied using the MTS Dermaroller. ${ }^{35}$

\subsection{Combination therapy:}

\section{A] Combination of Iontophoresis and Microneedles:}

In Iontophoresis a small electrical current is used for transportation of drug across the stratum corneum of the skin. The main advantage of using Iontophoresis along with microneedles is to control delivery of drug by controlling the current. The current may be turned on and off by the patient, and can deliver small drug molecules and bio molecules having a molecular weight up to a few thousand Daltons. ${ }^{36}$

\section{B] Combination of Electroporation and microneedles:}


Electroporation causes localized perturbation by forming aqueous pathways in the lipid bilayer of skin using high voltage short-duration current. A trans-membrane potential up to 1 $\mathrm{kV}$ for $10 \mathrm{~ms}$ to $500 \mathrm{~ms}$ was used for in-vitro Electroporation of stratum corneum. ${ }^{37}$ Longer pulse width and higher voltage was required to increase skin perturbation. This technique was also used for permeation enhancement of larger molecules having molecular weight up to several kilo Daltons. Furthermore, each microneedle behaved as a microelectrode for Electroporation, which eradicated the need for electrodes. Electroporation can be used in concert with chemotherapy (electrochemotherapy) for effective tumor treatment. Design of a silicon microneedle electrode array completed with integrated temperature and fluidic system for drug delivery specifically to tumor cells.

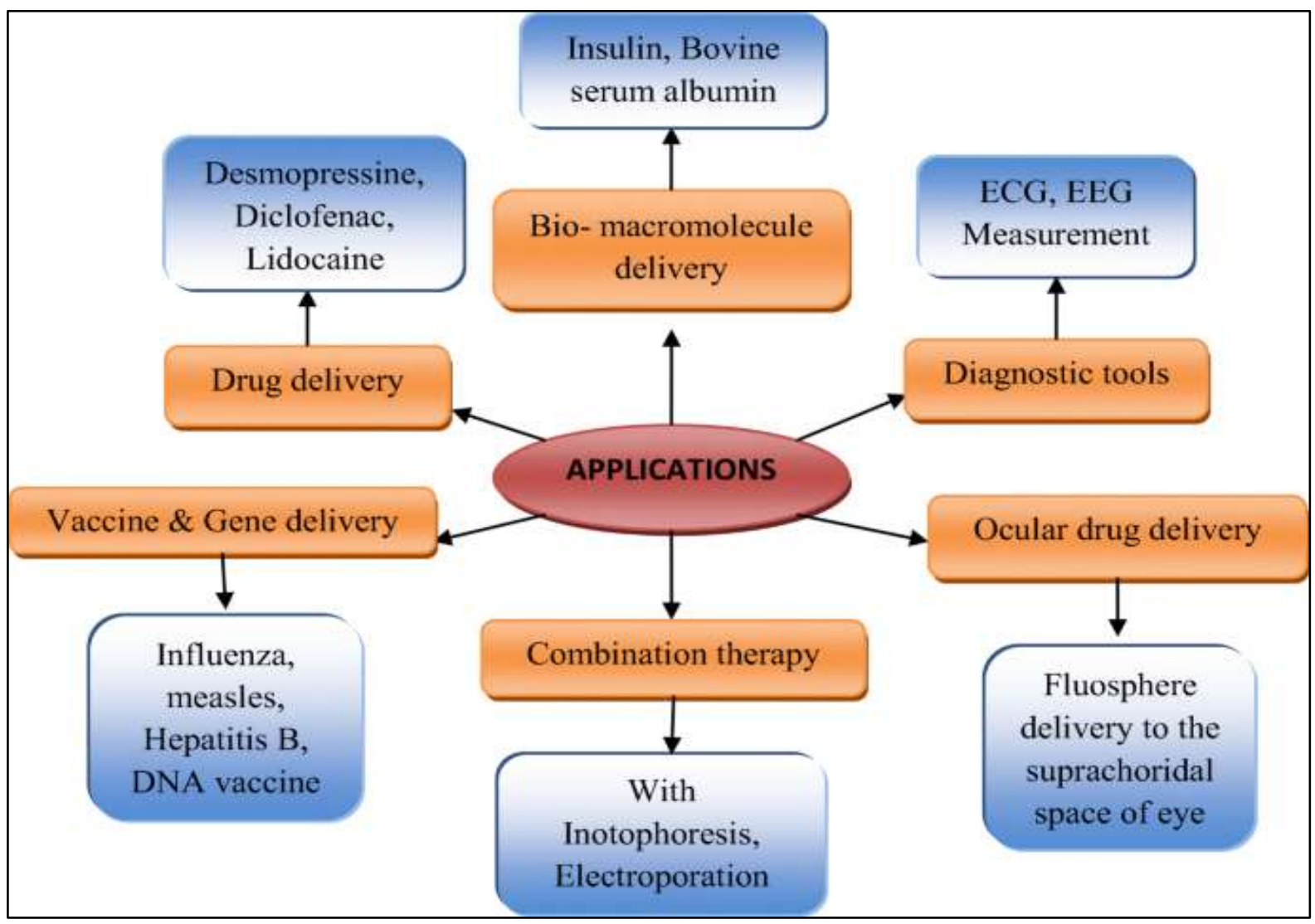

Figure No. 7 Applications of Microneedle Drug Delivery System

\section{Safety Issues of Microneedles:}

Microneedles have been used for the safe and effificient delivery of drugs and vaccines by creating reversible micro channels in the skin. Disruption of the stratum corneum by using conventional needles for the delivery of drugs and vaccines may cause pain, bleeding, skin irritation, skin redness and infection. The use of microneedles is considered safe owing to their small size and observed lack of significant damage to sensory neurons and blood vessels situated in the dermis layer beneath the skin, which means negligible pain and bleeding. But as microneedles disrupt the stratum corneum, safety concerns should be taken into account. Many studies related to the safety of microneedles have been conducted and these have proved their safety. Micro channels created by needles may cause chances of infection at the injection site because of permeation of pathogenic microbes or any toxic substances. Faster resealing of the micro channels is essential to prevent infection. In a study of the kinetics of 
skin resealing after insertion of microneedles, compared the time taken for skin resealing after application of microneedle and hypodermic needle (26 Gauge). ${ }^{38}$ They found that pain associated with needle insertion was significantly less with microneedles. Some transient reactions occurring on the skin at the site of microneedle application were found to be tolerable. They concluded that efficacious vaccination could be achieved using microneedles as compared with conventional intramuscular delivery of influenza vaccine. ${ }^{39}$

\section{Approved products:}

\begin{tabular}{|c|c|c|}
\hline Brand Name & Manufactured by & Applications \\
\hline VaxMat & TheraJect Inc., USA & $\begin{array}{l}\text { It is dissolvable microneedles and } \\
\text { can deliver hundreds of micrograms } \\
\text { of drug rapidly through the stratum } \\
\text { corneum into the epidermal tissue. }\end{array}$ \\
\hline Micro-Trans & Valeritas Inc., USA & $\begin{array}{l}\text { It can deliver the drug into dermis } \\
\text { without limitations of drug size, } \\
\text { structure, charge or the patient's skin } \\
\text { characteristics. }\end{array}$ \\
\hline Nanoject & Debiotech, Switzerland & $\begin{array}{l}\text { Useful for intradermal and } \\
\text { hypodermic drug delivery and for } \\
\text { interstitial fluid diagnostics. }\end{array}$ \\
\hline Janisys & Janisys, Ireland & $\begin{array}{c}\text { Actively delivers drugs from } \\
\text { transdermal patches and multiple } \\
\text { drugs can be administered via one } \\
\text { patch. }\end{array}$ \\
\hline BD Soluvia & Becton Dickinson, USA & $\begin{array}{l}\text { It is a prefillable microinjection } \\
\text { system for accurate intradermal } \\
\text { delivery of drugs and vaccines. }\end{array}$ \\
\hline Onvax & Becton Dickinson, USA & $\begin{array}{l}\text { It is a skin micro abrader having } \\
\text { plastic microneedles for disruption of } \\
\text { stratum corneum for the delivery of } \\
\text { vaccines. }\end{array}$ \\
\hline MicronJet & NanoPass Inc., Israel & $\begin{array}{l}\text { It can be used with any standard } \\
\text { syringe for painless delivery of } \\
\text { drugs, protein and vaccines approved } \\
\text { for this delivery route. }\end{array}$ \\
\hline
\end{tabular}

Table No. 1 List of approved products

\section{Conclusion :}

MNA have become a resource with great presence and prospects in the field of transdermal drug delivery. Their enormous potential lies in their ability to disrupt the barrier function exerted by the skin, and by doing so in a less invasive manner than other methods. The insertion of these devices does not reach the nervous terminations of the skin, making it a 
practically painless delivery method. On the other hand, it has been shown that the anxiety levels caused by MNA are considerably lower than those generated by other methods such as injections, which potentially would increase compliance rates, especially in the pediatric population. They can be used to achieve both local and systemic effects as they release the drug at the interphase of stratum corneum and viable epidermis. The types of molecules administered by these devices are varied, being of greatest interest those intended to exert systemic effects. For example, vaccination is a field where microneedles have great potential, since doses of antigen commonly administered are not very high. In this case, the use of MNA could be a much simpler and accessible approach to the general population, allowing mass vaccination campaigns for large populations.

\section{Reference:}

1. M. Rauma, A. Boman and G. Johanson, Adv. Drug Delivery Rev., 2013, 65, 306-314.

2. R. Wong, S. Geyer, W. Weninger, J. C. Guimberteau and J. K. Wong, Exp. Dermatol., 2016, 25, 92-98.

3. L. Gravitz, Nature, 2018, 563(7732), S83.

4. A. Menter, A. Gottlieb, S. R. Feldman, A. S. Van Voorhees, C. L. Leonardi, K. B. Gordon, M. Lebwohl, J. Y. Koo, C. A. Elmets, N. J. Korman, K. R. Beutner and R. Bhushan, J. Am. Acad. Dermatol., 2008, 58, 826-850.

5. Prausnitz, M.R. Engineering Microneedle Patches for Vaccination and Drug Delivery to Skin. Annu. Rev. Chem. Biomol. Eng. 2017, 8, 177-200.

6. Li, W.-Z.; Huo, M.-R.; Zhou, J.-P.; Zhou, Y.-Q.; Hao, B.-H.; Liu, T.; Zhang, Y. Super-Short Solid Silicon Microneedles for Transdermal Drug Delivery Applications. Int. J. Pharm. 2010, 389, 122-129

7. Gupta, J.; Gill, H.S.; Andrews, S.N.; Prausnitz, M.R. Kinetics of Skin Resealing after Insertion of Microneedles in Human Subjects. J. Control. Release 2011, 154, 148-155.

8. Kalluri, H.; Banga, A.K. Formation and Closure of Microchannels in Skin Following Microporation. Pharm. Res. 2011, 28, 82-94.

9. Donnelly, R.F.; Singh, T.R.R.; Alkilani, A.Z.; McCrudden, M.T.C.; O’Neill, S.; O’Mahony, C.; Armstrong, K.; McLoone, N.; Kole, P.; Woolfson, A.D. HydrogelForming Microneedle Arrays Exhibit Antimicrobial Properties: Potential for Enhanced Patient Safety. Int. J. Pharm. 2013, 45, 76-91.

10. Li, S.; Li, W.; Prausnitz, M. Individually Coated Microneedles for Co-Delivery of Multiple Compounds with Difffferent Properties. Drug Deliv. Transl. Res. 2018, 8, 1043-1052.

11. Koutsonanos, D.G.; del Pilar Martin, M.; Zarnitsyn, V.G.; Sullivan, S.P.; Compans, R.W.; Prausnitz, M.R.; Skountzou, I. Transdermal Inflfluenza Immunization with Vaccine-Coated Microneedle Arrays. PLoS ONE, 2009, 4, e4773.

12. Ito, Y.; Yoshimitsu, J.-I.; Shiroyama, K.; Sugioka, N.; Takada, K. Self-Dissolving Microneedles for the Percutaneous Absorption of EPO in Mice. J. Drug Target 2006, $14,255-261$.

13. Hong, X.; Wei, L.; Wu, F.; Wu, Z.; Chen, L.; Liu, Z.; Yuan, W. Dissolving and Biodegradable Microneedle Technologies for Transdermal Sustained Delivery of Drug and Vaccine. Drug Des. Devel. Ther. 2013, 7, 945-952. 
14. Li, W.; Terry, R.N.; Tang, J.; Feng, M.R.; Schwendeman, S.P.; Prausnitz, M.R. Rapidly Separable Microneedle Patch for the Sustained Release of a Contraceptive. Nat. Biomed. Eng. 2019, 3, 220-229.

15. Norman, J.J.; Choi, S.-O.; Tong, N.T.; Aiyar, A.R.; Patel, S.R.; Prausnitz, M.R.; Allen, M.G. Hollow Microneedles for Intradermal Injection Fabricated by Sacrifificial Micromolding and Selective Electrodeposition. Biomed. Microdevices 2013, 15, 203210.

16. Mikszta JA, Alarcon JB, Brittingham JM et.al. 2002. Improved genetic immunization via micromechanical disruption of skin barrier function and targeted epidermal delivery. Nat Med. 2002;8:415-419.

17. Cormier M, Johnson B, Ameri M, Nyam K, Libiran L, Zhang DD and Daddona P. Transdermal delivery of desmopressin using a coated microneedle array patch system. J Control Release. 2004;97(3):503-511

18. Langer R. 1998. Drug delivery and targeting. Nature 392(6679): 5-10 [Medline]

19. Langer R. New methods of drug delivery Science. 1990;49:1527-33.

20. Crystal RG. Transfer of genes to humans: early lessons and obstacles to success. Science. 1995; 0:404-10.

21. Davis SP et al. Insertion of microneedles into skin: measurement and prediction of insertion force and needle fracture force. J Biomech 2004; 37: 1155-1163.

22. Wang PC et al. Hollow polymer microneedle array fabricated by photolithography process combined with micro molding technique. Proceedings of Conference of Proc IEEE Eng Med Biol Soc 2009: 7026-7029.

23. Shawgo RS et al. BioMEMS for drug delivery. Curr Opin Solid State Mater Sci 2002; 6: 329-334.

24. Forvi E et al. A method to determine the margin of safety for microneedles arrays. Proceedings of the World Congress on Engineering 2010. 2, WCE 2010, June 30-July 2, 2010, London, 2010.

25. Wu Y et al. Microneedle-based drug delivery: studies on delivery parameters and biocompatibility. Biomed Microdevices 2008; 10: 601-610.

26. Teo MAet al. In vitro and in vivo characterization of MEMS microneedles. Biomed Microdevices 2005; 7: 47-52.

27. Hirschberg HJ et al. Bioneedles as alternative delivery system for hepatitis B vaccine. J Control Release 2010; 147: 211-217.

28. Verbaan FJ et al. Assembled microneedle arrays enhance the transport of compounds varying over a large range of molecular weight across human dermatomed skin. $J$ Control Release 2007; 117.

29. Donnelly RF et al. Microneedlemediated intradermal delivery of 5-aminolevulinic acid: potential for enhanced topical photodynamic therapy. J Control Release 2008; 129: 154-162.

30. Lo R et al. A passive MEMS drug delivery pump for treatment of ocular diseases. Biomed Microdevices 2009; 11: 959-970.

31. Smart WH, Subramanian K. The use of silicon microfabrication technology in painless blood glucose monitoring. Diabetes Technol Ther 2000; 2: 549-559.

32. Mukerjee EV et al. Microneedle array for transdermal biological fluid extraction and in situ analysis. Sens Actuators A Phys 2004; 114: 267- 275. 
33. Yuzhakov VV. Tissue conforming microneedle array and patch for transdermal drug delivery and biological flfluid collection. United States Patent 2010; US7,785,301 B2.

34. Mukerjee EV et al. Microneedle array for transdermal biological fluid extraction and in situ analysis. Sens Actuators A Phys 2004; 114: 267- 275.

35. How does 'Microneedle Therapy System' work? Clinical Resolution Laboratory, Inc., USA. http://www. microneedle.com/main/whatismts, accessed 12 October 2011).

36. Prausnitz MR, Langer R. Transdermal drug delivery.Nat Biotechnol 2008; 26: 12611268.

37. Naik A et al. Transdermal drug delivery: overcoming the skin's barrier function. Pharm Sci Technol Today 2000; 3: 318-326.

38. Gupta Jet al. Kinetics of skin resealing after insertion of microneedles in human subjects. J Control Release 2011; 154: 148-155.

39. Van Damme P et al. Safety and efficacy of a novel microneedle device for dose sparing intradermal influenza vaccination in healthy adults. Vaccine 2009; 27: 454459. 